\title{
Proficient Character Recognition from Images
}

\author{
Poornima T. M. \\ Final Year-B.Tech. \\ (Information Technology) \\ Aalim Muhammed Salegh \\ College of Engineering \\ Chennai-55
}

\author{
M. Amanullah, $\mathrm{PhD}$ \\ HOD - Dept. Of Information Technology \\ Aalim Muhammed Salegh \\ College of Engineering \\ Chennai-55
}

\begin{abstract}
Reading text from photographs is a challenging problem that has received a significant amount of attention. Two key components of most systems are (i) text detection from images and (ii) text recognition, and many methods have been introduced to design better feature representations and models for both. Scene text recognition has gained significant attention from the computer vision community in recent years. Recognizing text is a difficult problem, even more so than the detection of scanned documents. To evaluate the performance of recent algorithms in detecting and recognizing text from complex images, In this proposed paper implement two method text detection and text recognition. The features extractors are Harris-Corner, Maximal Stable Extremal Regions (MSER), and dense sampling and Histogram of Oriented Gradients (HOG) descriptors. Then implement text recognition. The first one is training a character recognizer to predict the category of a character in an image patch. The second one is training a binary character classifier for each character class to predict the existence of this category in an image patch. The two schemes are compatible with two promising applications related to scene text, which are text understanding and text retrieval. Further we extend this concept with word level recognition with lexicon techniques with accurate results. And also recognition text in real time images, videos and mobile application images.
\end{abstract}

\section{Keywords}

Character Recognition, Text Detection, Text Recognition

\section{INTRODUCTION}

Investigate in archive investigation and distinguishment has generally centered around transforming and examining checked records. Locating content in characteristic pictures, as distinctive to filtering of printed pages, faxes and business cards, is a principle venture for a measure of Computer Vision applications, for example, computerized help for outwardly debilitated, normal geocoding of organizations, and automated guide perusing in urban situations. Versatile visual hunt has picked up prevalent enthusiasm with the expanding accessibility of superior, ease cam telephones. Lately, picture look frameworks have been created for applications, for example, item distinguishment and milestone distinguishment .In these cases, content can be concentrated as an abnormal state peculiarity to supplement low-level visual peculiarities in substance based picture/feature recovery frameworks. It can likewise be utilized within an extensive variety of other mixed media applications, for example, versatile picture hunt, sign change and business name examine in road level pictures. The challenges originate from the way that characters installed in the scene can show up in a few textual styles, with a few shades, and on muddled foundations. Common scene showcase board pictures contain content data which is regularly needed to be naturally perceived and process. Scene content may be any literary piece of the standpoint pictures, for example, names of roads, foundations names, names of shops, development names, organization names, road signs, movement data, notice signs and so forth. Analysts have cautioned their consideration on advancement of strategies for comprehension message on such show sheets. Msers mean a set of recognized locales, which are characterized by an extremal property of its force work in the locale and on its external limit. What's more, Msers have all the properties needed of a stable neighborhood identifier. As of late, Maximally Stable Extremal Regions (Msers) based content recognition has been broadly investigated. The principle point of interest of these methodologies over other segment based methodologies is established in the viability of utilizing Msers as character/part applicants. It is focused around the perception that content segments normally have higher shade stand out from their experiences and have a tendency to be structure homogenous color areas, at any rate at the character level. The MSER calculation adaptively recognizes stable shade locales and gives a decent answer for restrict the segments without express binarization. Content location and distinguishment in regular scene pictures has as of late gotten expanded consideration of the machine vision group. Since content is a pervasive component in numerous situations, tackling this issue has potential for huge effect.

\section{RELATED WORK}

\section{A. X. Bai, L. J. Latecki, and W.-Y. Liu et al[1]}

In this paper, we present another skeleton pruning technique focused around form apportioning. Any shape parcel can be utilized, however the parcels acquired by Discrete Curve Evolution (DCE) yield phenomenal results. The hypothetical properties and the analyses introduced exhibit that got skeletons are as per human visual recognition and steady, even in the vicinity of critical commotion and shape varieties, and have the same topology as the first skeletons. Specifically, we have demonstrated that the proposed approach never creates spurious extensions, which are regular when utilizing the known skeleton pruning routines. Also, the proposed pruning system does not dislodge the skeleton focuses. Thus, all skeleton focuses are focuses of maximal circles. Once more, numerous existing routines uproot skeleton indicates in place produces pruned skeletons.

\section{B. R. Beaufort and C. Mancas-Thillou [2]}

With the expanding business sector of shoddy cams, common scene content must be taken care of in a proficient way. A few works manage content recognition in the picture while later ones call attention to the test of content extraction and distinguishment. We propose here an OCR revision framework to handle customary issues of recognizer lapses additionally the ones because of common scene pictures, i.e. cut characters, creative presentation, fragmented sentences (display in promotions) and out- of-vocabulary (OOV) words, for example, acronyms etc. The principle calculation bases on 
limited state machines (Fsms) to manage learned OCR disarrays, capital/accentuated letters and dictionary look-up. In addition, as OCR is not considered as a discovery, a few yields are considered to mix distinguishment and rectification steps. In light of an open database of characteristic scene words, point by point results are additionally exhibited alongside future works.

\section{X. Chen, J. Yang, J. Zhang, and A. Waibel, et al [3]}

In this paper, we display a methodology to programmed location and distinguishment of signs from characteristic scenes, and its application to a sign interpretation assignment. The proposed methodology implants multiresolution and multiscale edge location, versatile looking, color investigation, and relative correction in a progressive system for sign recognition, with distinctive attentions at each one stage to handle the content in diverse sizes, introductions, shade disseminations and foundations. We utilize relative amendment to recoup disfigurement of the content areas brought on by an improper cam perspective point. The methodology can essentially enhance content discovery rate and optical character distinguishment (OCR) precision. As opposed to utilizing parallel data for OCR, we concentrate characteristics from a force picture straightforwardly. We propose a neighborhood force standardization technique to successfully handle lighting varieties, took after by a Gabor change to get nearby gimmicks, lastly a direct separate dissection (LDA) strategy for peculiarity choice. We have connected the methodology in creating a Chinese sign interpretation framework, which can consequently locate and perceive Chinese signs as information from a cam, and make an interpretation of the perceived content into English.

\section{STROKE WIDTH TRANSFORM}

A stroke in the picture is a continuous band of an about consistent width. The methodology of SWT is gathering pixels together in a snappy path, as an option of searching for removal peculiarities of pixels. The Stroke Width Transform (SWT) is a nearby administrator which figures for every pixel the width of the in all likelihood stroke containing the pixel. SWT tries to catch the main content viable gimmicks and utilizing geometric signature of content to channel out nonmessage regions. As a result, SWT provides for you predictable content areas that is dialect nonpartisan.

\section{Advantages}

- One of the quick techniques.

- Reliable and adaptable strategy for content recognition.

E. Disadvantage

- This is not considering the heading of stroke so bended content line not discovered.

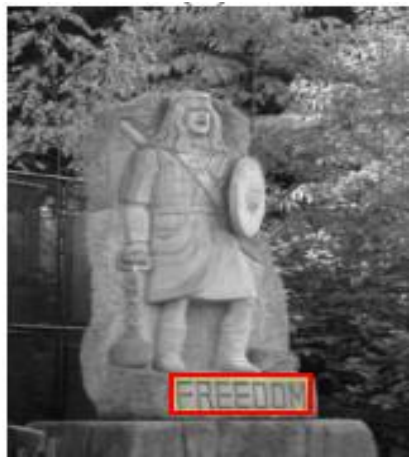

Figure 1: SWT Detect the Text in the Image

\section{CHAOS GENETIC FEATURE SELECTION OPTIMIZATION}

The hereditary calculation fits in with the group of evolutionary registering as a helpful device for hunt advancement issues, alongside hereditary programming, improvement procedures, and evolutionary preparing. The hereditary calculation is a versatile heuristic inquiry calculation determined from the idea of regular choice and characteristic hereditary qualities. The saying "hereditary qualities" is determined from the Greek word "genesis" signifying "to develop". It is the extension of science that separates in the middle of heredity and varieties and record for the likenesses and contrasts amid the procedure of advancement. "Bedlam is a name for any request that delivers perplexity in our personalities" Chaos hypothesis is an extension in math applications in different teaches, for example, physical science, designing, trading and lending, science and logic. In like manner use, "bedlam" signifies "A condition or spot of incredible issue or puzzlement". Disorder hypothesis ponders the execution of frameworks that take after deterministic laws yet seem irregular and capricious or we can say a dynamical framework that has a touchy reliance on its introductory conditions; little changes in those conditions can prompt very distinctive conclusions.

\section{F. Advantages}

- More extensive arrangement space.

- Intricacy in the wellness scene

\section{G. Disadvantage}

- One of the detriments is 'Untimely merging' that is for Random reason.

\section{FEATURE LEARNING ALGORITHM}

Peculiarity learning or representation learning is a situated of systems in machine discovering that take in a change of "crude" inputs to a representation that can be adequately misused in an administered learning errand, for example, classification. Gimmick learning calculations themselves may be unsupervised or regulated, and incorporate auto encoders, lexicon learning, lattice factorization, confined Boltzmann machines and different manifestations of bunching.

\section{H. Advantages}

- Encouraging data disclosure and information understanding.

- Dropping the estimation and capacity necessities

\section{Disadvantage}

- Free Component Analysis a disservice contrasted with different strategies.
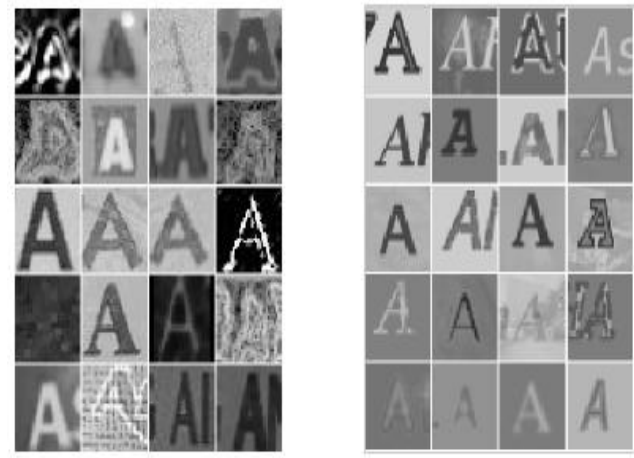

Figure2: Training examples 


\section{OPTICAL CHARACTER RECOGNITION}

OCR is optical character recognition, a product instrument that permits trading filtered record into content searchable records. It is currently continuously more basic for archives to be filtered so they can be effectively seen and imparted through electronic means. A sweep is simply a picture catch of the one of a kind archive, so it can't be abbreviated or looked all through in any capacity. This results in a decrease in productivity since workers now need to physically correct or seek through various pages. OCR tackles this inconvenience by making the archive content searchable.

\section{J. Advantages}

- It builds the viability and estimation of office work.

- The capacity to quickly seek through substance is hugely helpful, essentially in an office setting that need to orchestrate with high volume examining or high archive inflow

\section{HARRIS CORNER DETECTOR}

A corner can be characterized as the crossing point of two edges or a point. It is intersections of shapes. For the most part corner focuses are more steady peculiarities over progressions of perspective. Corner location is broadly utilized within machine vision application, for example, movement recognition, picture matching, following. In this module, Harris corner locator is utilized to concentrate the corner focuses. The Harris corner locator is a prevalent investment point identifier. Since there is no impact of revolution, scale, enlightenment variety, and picture commotion on the execution of Harris corner finder. It is based upon the nearby auto-relationship capacity of a sign, where the neighborhood auto-correspondence capacity measures the nearby changes of the sign with patches moved by a little sum in distinctive bearings.

\section{K. Advantages}

- Immediate system utilizes the greater part of the accessible information and can give extremely precise enlistment. This is the preference of it.

- No clamor affectability deduction or other computationally lavish operations must be performed.

\section{EXTREMAL REGIONS DETECTOR}

Another set of picture components that are put into correspondence, the supposed extremal locales. Extremal districts have exceedingly attractive properties: the set is shut under 1. Ceaseless change of picture facilitates 2. Monotonic change of picture splendor. Discovering extremal areas: locate stay focuses.Anchor focuses recognized at various scales are nearby extremas of power .Explore picture around beams from each one grapple point. Come each beam beginning starting here until an extremum of capacity $f$ is arrived at. All focuses make some eccentrically formed area. Give or take comparing areas are gotten for relative changed districts.

\section{Advantages}

- The speedup of the processing time and the change of the location and following dependability are assessed.

- The edge-upgraded MSER discovered in the inquiry picture can be utilized to concentrate characteristic descriptors like for visual pursuit.

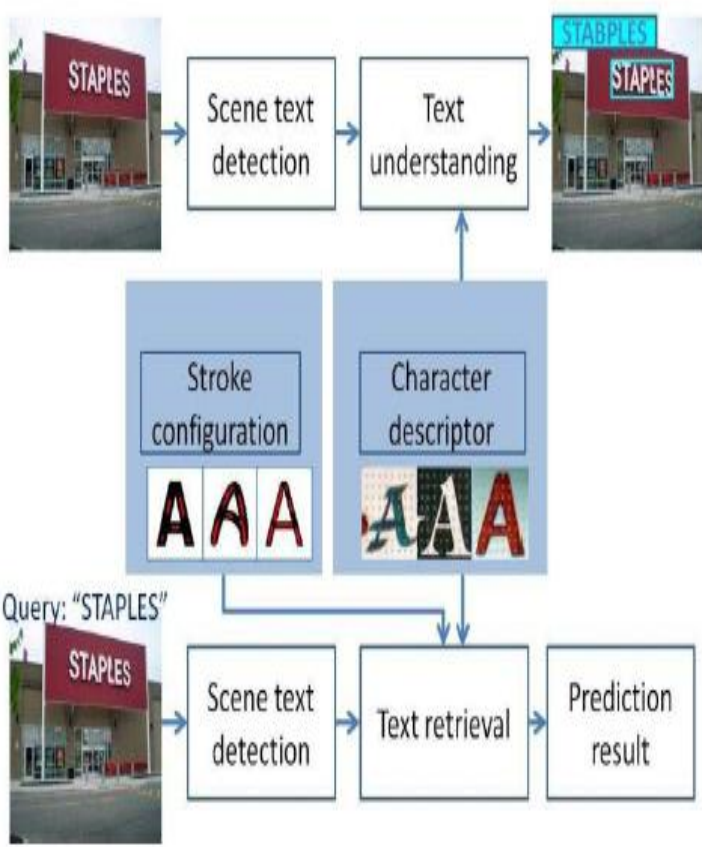

Figure 3: Flowchart of text extraction method

\section{EXPERIMENTAL RESULTS}

In trial results demonstrate the precision rate of content location by contrasting stroke width change, Chaos Genetic Feature Selection Optimization, MSER, Feature Learning calculation and proposed strategy.

Table 1: Accuracy result comparison Table

\begin{tabular}{|c|c|c|}
\hline Algorithm & AR & FPR \\
\hline SWT & 0.56 & 0.9 \\
\hline Chaos & 0.45 & 0.6 \\
\hline OCR & 0.67 & 0.7 \\
\hline Feature Learning & 0.34 & 0.8 \\
\hline Proposed & 0.89 & 0.4 \\
\hline
\end{tabular}

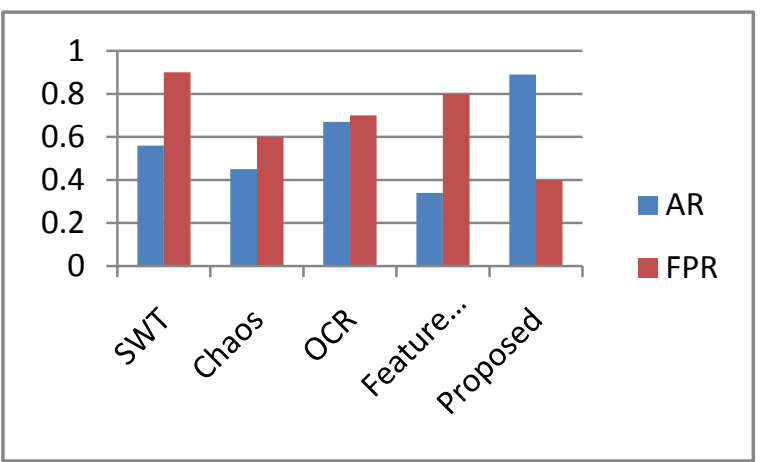

Figure 4: Accuracy comparison of algorithm

\section{CONCLUSION}

In this proposed a Scene content recognition and scene content distinguishment framework connected to pictures to catch the content in the picture. The proposed procedure is contrasts with different peculiarities how strategy is superior to the next method. It is perfect with versatile applications. It identifies the content from the regular picture or a feature from the 
perceived content districts. To hunt down a picture locales of content strings by utilizing the color disintegration and level arrangement. There are two plans in content distinguishment content comprehension and content recovery. In future work, enhance the precision rate of content recognition, and add dictionary dissection to expand our framework to word-level distinguishment.

\section{REFERENCES}

[1] X. Bai, L. J. Latecki, and W.-Y. Liu, "Skeleton pruning by contour partitioning with discrete curve evolution," IEEE Trans. Pattern Anal. Mach. Intell., vol. 29, no. 3, pp. 449-462, Mar. 2007.

[2] R. Beaufort and C. Mancas-Thillou, "A weighted finitestate framework for correcting errors in natural scene OCR," in Proc. 9th Int. Conf. Document Anal. Recognit., Sep. 2007, pp. 889-893.

[3] X. Chen, J. Yang, J. Zhang, and A. Waibel, "Automatic detection and recognition of signs from natural scenes," IEEE Trans. Image Process., vol. 13, no. 1, pp. 87-99, Jan. 2004.

[4] A. Coates et al., "Text detection and character recognition in scene images with unsupervised feature learning," in Proc. ICDAR, Sep. 2011, pp. 440-445.
[5] N. Dalal and B. Triggs, "Histograms of oriented gradients for human detection," in Proc. IEEE Conf. Comput. Vis. Pattern Recognit., Jun. 2005, pp. 886-893.

[6] T. de Campos, B. Babu, and M. Varma, "Character recognition in natural images," in Proc. VISAPP, 2009.

[7] B. Epshtein, E. Ofek, and Y. Wexler, "Detecting text in natural scenes with stroke width transform," in Proc. CVPR, Jun. 2010, pp. 2963-2970.

[8] P. F. Felzenszwalb, R. B. Girshick, D. McAllester, and D. Ramanan, "Object detection with discriminatively trained part-based models,"IEEE Trans. Pattern Anal Mach. Intell., vol. 32, no. 9, pp. 1627-1645, Sep. 2010.

[9] T. Jiang, F. Jurie, and C. Schmid, "Learning shape prior models for object matching," in Proc. CVPR, Jun. 2009, pp. 848-855.

[10] S. Kumar, R. Gupta, N. Khanna, S. Chaudhury, and S. D. Johsi, "Text extraction and document image segmentation using matched wavelets and MRF model," IEEE Trans. Image Process., vol. 16, no. 8, pp. 2117 2128, Aug. 2007. 